\title{
Diabetes mellitus, insulin resistance, and metabolic syndrome in HIV-positive patients in South India
}

This article was published in the following Dove Press journal:

International Journal of General Medicine

21 January 2011

Number of times this article has been viewed

\author{
Jyothi Idiculla' \\ G D Ravindra'n' \\ Jason D'Souza' \\ Girija Singh' \\ Sultana Furruqh ${ }^{2}$ \\ 'Department of Medicine, \\ 2Department of Biochemistry, \\ St. John's Medical College, Bangalore, \\ India
}

\begin{abstract}
Insulin resistance, diabetes mellitus, and metabolic syndrome in patients with human immunodeficiency virus (HIV) infection are increasingly being reported in the global medical literature. This cross-sectional study was done to describe the occurrence of metabolic syndrome, diabetes mellitus, and insulin resistance in HIV-positive patients in a tertiary referral center in South India. A total of 60 patients who had HIV infection for 12 months or more were enrolled in the study. Of these, 30 patients were antiretroviral therapy (ART)-naïve, and 30 were treated with ART. Biochemical estimations (fasting blood glucose, $75 \mathrm{~g}$ oral glucose tolerance test, lipid profile, and fasting insulin) and anthropometric measurements (height, weight, and waist circumference) were performed for each patient. Metabolic syndrome was diagnosed using National Cholesterol Education Program-Adult Treatment Plan III criteria, and insulin resistance was calculated applying the homeostasis model assessment method. Diabetes mellitus, impaired fasting glycemia, and impaired glucose tolerance were diagnosed based on American Diabetes Association criteria. A high prevalence of metabolic syndrome was observed in patients with HIV (16/60), and was more prevalent in the ART-treated group $(13 / 30 ; P=0.028)$. Similarly, insulin resistance was also noted to be high $(24 / 60)$, and of these patients, 15 were on ART. Seventy-five percent of patients with metabolic syndrome had insulin resistance. Diabetes was diagnosed in one patient who was ART-naïve and in six patients who were on ART. Our observations suggest an increased prevalence of metabolic syndrome, insulin resistance, and diabetes mellitus in ART-treated patients. These warrant attention and substantiation with larger studies. While ART improves survival, it may lead on to cardiovascular morbidity and mortality, especially in the Indian subcontinent where there is a genetic predisposition to cardiovascular risk.
\end{abstract}

Keywords: human immunodeficiency virus (HIV) infection, metabolic syndrome, insulin resistance, diabetes mellitus

\section{Introduction}

Human immunodeficiency virus (HIV), which was first discovered in 1981, has now infected more than two million people in India. The introduction of antiretroviral therapy (ART) has resulted in significant reductions in mortality and morbidity of these patients. However, the occurrence of insulin resistance and metabolic syndrome has been reported as a side effect of this therapy. ${ }^{1}$ The drugs implicated include nucleoside reverse transcriptase inhibitors (major components of first-line therapy in India) and also protease inhibitors (components of current second-line therapy in India). With alteration in insulin sensitivity, dysglycemia, dyslipidemia, hypertension, and body fat abnormalities, there are concerns about the long-term risks of ART. ${ }^{2}$ In India,
Correspondence: Jyothi Idiculla Department of Medicine, St. John's Medical College, Sarjapur Road, Bangalore 560034, India

Email jyothi_idiculla@yahoo.co.in 
where there is genetic predisposition to insulin resistance and cardiovascular risk, ${ }^{3,4}$ this impact may be significant. Hence, this study was undertaken to assess the prevalence of these conditions in HIV-infected patients.

The aims of the study were to: 1) estimate the prevalence of metabolic syndrome, insulin resistance, and diabetes mellitus in HIV-infected patients on ART; and 2) compare the prevalence of these conditions between patients who are on ART and those who are ART-naïve.

\section{Methods}

This is a cross-sectional study involving HIV-positive patients conducted in a tertiary referral centre in Bangalore. Patients were enrolled into the study if they were aged over 18 years, had HIV infection for more than 12 months, and were on ART (ART-treated group) or had HIV infection for more than 12 months but were not on ART (ART-naïve group). Patients were not included in the study if diabetes mellitus, hypertension, dyslipidemia, coronary artery disease, malignancy, sleep apnea, or chronic renal failure were present prior to starting ART or if they were nonadherent to ART.

Patients who satisfied the criteria were informed of the study and its details, following which a consent form was signed. The demographic details were collected from each patient and entered into the pro-forma. Subsequent to this, a detailed history was taken, and a thorough physical examination was done. The anthropometric measurements were done by a skilled nutritionist, and these were height, weight, and waist circumference, measured using height scale, weighing machine, and inch tape, respectively, standardized regularly in the St. John's Medical College Nutrition Research Centre. Height was recorded to the nearest centimeter, and weight was recorded to the nearest $0.5 \mathrm{~kg}$. Waist girth was measured at the point between the iliac crest and the lower margin of the ribs and rounded to the nearest centimeter.

After an overnight fast, blood samples were drawn from each patient. Following this, $75 \mathrm{~g}$ glucose was administered, and at 2 hours another blood sample was drawn. Blood glucose levels, both fasting and post glucose load, were estimated using the hexokinase (enzymatic) method spectrophotometrically in $\mathrm{R} \times \mathrm{L}$ dimension automated equipment. The total cholesterol was determined using the cholesterol oxidase and cholesterol esterase method. High-density lipoprotein (HDL) cholesterol was estimated by the automated enzymatic method. Triglycerides were determined using the lipoprotein lipase/glucokinase enzymatic procedure. The lipid profile was estimated using Dade Behring equipment.
Fasting insulin levels were done using chemiluminescence method on immulite equipment from Diagnostics Product Corporation (Kolkata, India). All biochemical procedures were done in accordance with the International Federation of Clinical Chemistry (IFCC) quality control. The lab is under internal and external quality control and is National Accreditation Board for Testing and Calibration Laboratories (NABL)-accredited. CD-4 counts were done in the department of microbiology or private labs.

Metabolic syndrome was defined using National Cholesterol Education Program-Adult Treatment Plan III (NCEP-ATP III) 2001 criteria. $^{5}$ If three or more of the following criteria were present, metabolic syndrome was diagnosed:

- Waist circumference more than $102 \mathrm{~cm}$ in men and $88 \mathrm{~cm}$ in women,

- Triglycerides more than $150 \mathrm{mg} / \mathrm{dL}$,

- HDL cholesterol less than $40 \mathrm{mg} / \mathrm{dL}$ in men and less than $50 \mathrm{mg} / \mathrm{dL}$ in women,

- Blood pressure more than or equal to $130 / 85 \mathrm{~mm} \mathrm{Hg}$,

- Fasting glucose more than $110 \mathrm{mg} / \mathrm{dL}$.

Insulin resistance (IR) was calculated using the homeostatic model assessment of insulin resistance (HOMA-IR). This was obtained using the formula: ${ }^{6}$

$\underline{\text { Fasting insulin level (micro IU) } \times \text { fasting glucose }(\mathrm{mmol} / \mathrm{l})}$ 22.5

A cutoff value of 1.93 was taken as IR, based on the Chennai Urban Population Study. ${ }^{?}$

\section{Diabetes mellitus, impaired fasting glucose, and impaired glucose tolerance}

The following criteria were used to classify hyperglycemia: ${ }^{8}$

- Fasting plasma glucose $(\mathrm{FPG})<100 \mathrm{mg} / \mathrm{dL}(5.6 \mathrm{mmol} / \mathrm{L})=$ normal fasting glucose,

- FPG 100-125 mg/dL (5.6-6.9 mmol/L) = impaired fasting glucose (IFG),

- $\mathrm{FPG} \geq 126 \mathrm{mg} / \mathrm{dL}(7.0 \mathrm{mmol} / \mathrm{L})=$ provisional diagnosis of diabetes.

The corresponding categories when the oral glucose tolerance test (OGTT) is used are the following:

- Two-hour postload glucose $<140 \mathrm{mg} / \mathrm{dL}(7.8 \mathrm{mmol} / \mathrm{L})=$ normal glucose tolerance,

- Two-hour postload glucose 140-199 mg/dL $(7.8-11.1 \mathrm{mmol} / \mathrm{L})=$ IGT (impaired glucose tolerance),

- Two-hour postload glucose $\geq 200 \mathrm{mg} / \mathrm{dL}(11.1 \mathrm{mmol} / \mathrm{L})=$ provisional diagnosis of diabetes. 


\section{Ethics}

Approval from the Institutional Ethics Review Board was obtained prior to commencement of the study. All patients were provided with details of the aims of the study and the procedure following enrollment. A consent form was signed by all patients who were included in the study.

\section{Statistics}

The data were analyzed using SPSS software (version 16; SPSS Inc., Chicago, IL). Student's $t$-test was done to compare numerical variables in the two groups. Chi square test or Fisher's exact test were used for ordinal variables.

\section{Results}

A total of 60 patients were enrolled in the study. Of these, 30 patients were ART-naïve, and 30 were treated with ART. The mean duration of ART was 41.5 months (range 12-108 months). There were 23 males and seven females in the ART-treated group and 21 males and nine females in the ART-naïve group. The mean age of patients in the ARTtreated group was $41.63( \pm 10.94)$, while that in the ART group was $40.6( \pm 9.48)$. The mean duration of disease (HIV) was $53.3( \pm 27.76)$ months in the ART-treated group and $17.20( \pm 9.18)$ months in the ART-naïve group. Table 1 summarizes demographic details, components of metabolic syndrome, insulin resistance, and CD-4 counts in the ARTtreated and ART-naïve groups.

Applying NCEP-ATP III criteria, a total of 16 patients out of 60 were diagnosed to have metabolic syndrome. Of these, 13 (81.3\%) were on ART, while three (18.7\%) were not treated with ART $(P=0.028)$. Of the 60 patients, all had at least one component of metabolic syndrome. Four patients fulfilled four criteria, and all of them were on ART. The details of the number of patients with combinations of criteria are demonstrated in Figure 1. The majority of patients with metabolic syndrome were males (12/16). When the patients with metabolic syndrome were grouped into two, based on age (age $\leq 40$ years and age $>40$ years), nine were aged 40 years or younger. Four patients with metabolic syndrome had body mass index (BMI) less than 18.5, 10 had BMI ranging from 18.5 to 24.9, and two had BMI more than 25. There was none in the obese range with BMI above 30. Among the parameters used for diagnosis of metabolic syndrome, the most observed was low HDL, occurring in all patients. The details of distribution of individual components in patients with metabolic syndrome are graphically represented in Figure 2.

For evaluating the occurrence of metabolic syndrome with respect to the type of ART, the patients were classified into three groups: 1) nonprotease inhibitor (PI), nonstavudine (d4T), 2) non-PI d4T, and 3) PI-based regimens. Of the 30 patients in the ART group, three were exposed to PIs, 18 to $\mathrm{d} 4 \mathrm{~T}$, and nine received the non-PI, non-d4T regime. All three patients exposed to PI had metabolic syndrome. In the non-PI d4T group, 6 out of 18 had metabolic syndrome, and in the non-PI, non-d4T group 4 out of 9 had metabolic syndrome.

IR was detected in 24 (40\%) patients in the study group with a cutoff value of 1.93 for HOMA. Of these, $15(62.5 \%)$ were on ART, while nine $(37.5 \%)$ were ART naïve $(P=0.11)$. Thirteen were aged 40 years or less, whereas the rest were

Table I Comparison between ART-treated and ART-naïve groups

\begin{tabular}{|c|c|c|c|c|c|}
\hline \multirow[t]{2}{*}{ Study characteristic } & \multicolumn{2}{|c|}{$\operatorname{ART}(n=30)$} & \multicolumn{2}{|c|}{ ART-naïve $(n=30)$} & \multirow[t]{2}{*}{$P$ value } \\
\hline & Mean & SD & Mean & SD & \\
\hline Age (years) & 41.63 & 10.94 & 40.60 & 9.48 & 0.697 \\
\hline Duration of disease (months) & 53.03 & 27.76 & 17.20 & 9.18 & $<0.001$ \\
\hline Male/female & $23 / 7$ & - & $21 / 9$ & - & - \\
\hline BMI $\left(\mathrm{kg} / \mathrm{m}^{2}\right)$ & 20.27 & 3.82 & $|8.4|$ & 4.27 & $0.080+$ \\
\hline Waist circumference $(\mathrm{cm})$ & 82.7 & 11.88 & 76.7 & 9.64 & 0.036 \\
\hline Systolic BP (mm Hg) & 125.67 & $|8.5|$ & 114.13 & 11.60 & 0.005 \\
\hline Diastolic BP (mm Hg) & 80.60 & 9.13 & 75.60 & 8.23 & 0.030 \\
\hline Fasting blood glucose (mg/dL) & 95 & 25.16 & 89.53 & 19.6 & 0.352 \\
\hline Two-hour OGTT blood glucose (mg/dL) & 111.33 & 58.47 & 131.33 & 28.55 & 0.094 \\
\hline HOMA & 2.50 & 2.68 & 1.80 & 1.93 & 0.265 \\
\hline Total cholesterol (mg/dL) & 157.47 & 43.58 & 149.17 & 39.92 & 0.445 \\
\hline HDL cholesterol (mg/dL) & 32.87 & 13.27 & 25.53 & 9.42 & 0.017 \\
\hline LDL cholesterol (mg/dL) & 86.92 & 32.91 & 78.50 & 21.63 & 0.281 \\
\hline Triglycerides (mg/dL) & 201.37 & 163.66 & 155.43 & 70.14 & 0.163 \\
\hline CD-4 counts (cells/ $\mu \mathrm{L})$ & 210.23 & 172.89 & 170.67 & 131.40 & 0.322 \\
\hline
\end{tabular}

Abbreviations: ART, antiretroviral therapy; BMI, body mass index; BP, blood pressure; HDL, high-density lipoprotein; HOMA, homeostasis assessment; LDL, low-density lipoprotein; OGTT, oral glucose tolerance test; SD, standard deviation. 


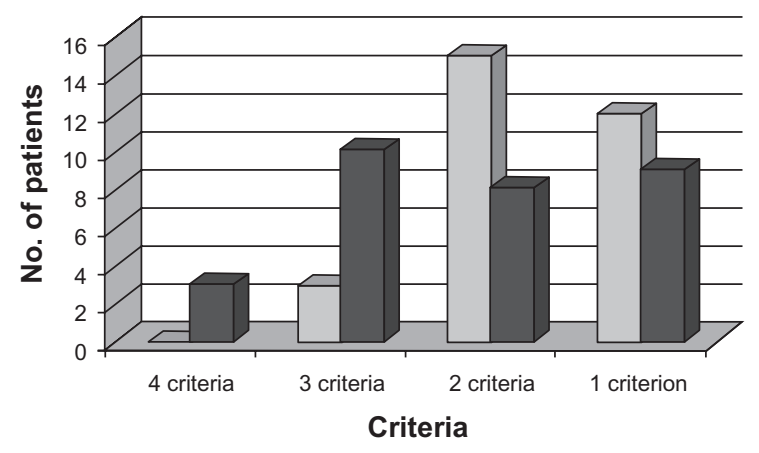

Figure I Numbers of patients versus criteria for metabolic syndrome. Notes: ART-naïv; $\square$ ART-treated.

over this age. Eighteen patients with IR were male, and six female. In patients with IR, BMI in seven patients was less than 18.5, and in 13 patients, between 18.5 and 24.9. Two patients with IR had BMI in the 25.0-29.9 range, and two were obese with BMI over 30. IR was present in 12 out of 16 patients $(75 \%)$ with metabolic syndrome. Looking at various treatment regimens, all three patients in the PI group had IR. In the d4T group, seven out of 18, and in the non-d4T group, five out of nine had IR.

In the ART-naïve group, five patients had IFG, two had IGT, and two had diabetes. In the ART-treated group, three had IFG, two had IGT, two had both IFG and IGT, and six had diabetes.

\section{Discussion}

The results of the study show that there is an increased prevalence of metabolic syndrome and IR in patients treated with ART. In our study, the overall prevalence of metabolic syndrome in HIV-positive patients was $26.6 \%$; $43.3 \%$ in the ART-treated group, and $10 \%$ in the ARTnaïve group. These results showed a statistically significant increase in the ART group $(P=0.028)$. Studies from Spain and other parts of the world have reported prevalence rates of $17 \%-18 \%$ on larger HIV populations using NCEP-ATP III criteria. ${ }^{9}{ }^{10}$ Indian studies on uninfected individuals have reported prevalence rates ranging from $18.4 \%$ to $30.9 \%$ using NCEP-ATP III criteria. ${ }^{11,12}$ Thus, in our study the overall prevalence rate appears to be similar to the Indian general population but higher than other HIV populations. The prevalence in our ART-treated group is much higher at $43.3 \%$. Insulin resistance based on HOMA values was present in $75 \%$ of our patients with metabolic syndrome, thereby reiterating its pivotal role in its pathogenesis of this condition.

It is of interest that all the patients included in the study had at least one criterion for metabolic syndrome, and the most observed was low HDL. In the Spanish study, of the total 710 study patients (both ART-treated and -naïve), one or more criteria were fulfilled by $69.3 \%$, two or more by $35.8 \%$, three or more by $17 \%$, four or more by $4.5 \%$, and all five criteria were seen only in one patient. ${ }^{9}$ Though not directly comparable, these results suggest that unlike in our patients, $31.7 \%$ did not have any feature of metabolic syndrome, implying that these metabolic derangements may be more prevalent in the Indian population. In our study, low HDL cholesterol was the most observed parameter, whereas in the Spanish study, hypertriglyceridemia was the most commonly recognized. Despite this difference, the dyslipidemia observed in our patients is

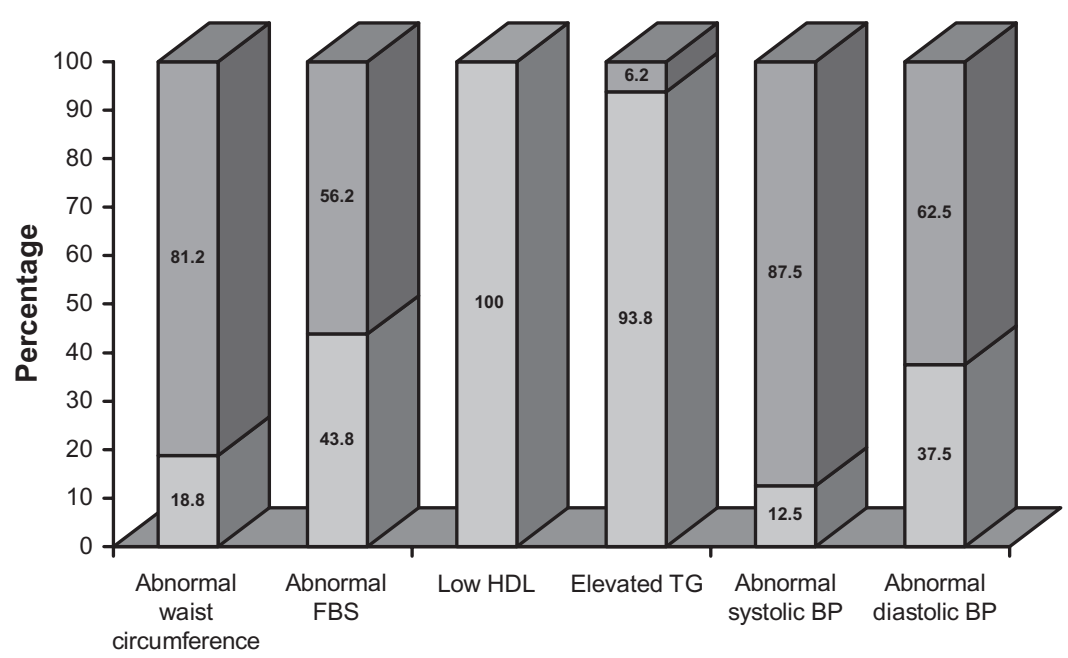

Parameters

Figure 2 Distribution of criteria among patients with metabolic syndrome.

Notes: parameter present; 1 parameter absent.

Abbreviations: BP, blood pressure; FBS, fasting blood sugar; HDL, high-density lipoprotein; TG, triglycerides. 
concordant with HIV associated dyslipidemia and is a confirmed risk factor for macrovascular disease. ${ }^{13}$

The mechanism of IR and metabolic syndrome in nucleoside reverse transcriptase inhibitor-treated patients is postulated to be mitochondrial toxicity. ${ }^{14}$ It should be kept in mind here that affluent nations have moved on to less toxic drugs, while developing countries like India still administer these drugs as part of national treatment programs. ${ }^{15}$ The individual contributions of drugs could not be assessed in our study due to inadequate numbers of patients, though 10 out of 27 patients on nucleoside reverse transcriptase inhibitor-based regimes had metabolic syndrome, and 12 out of 27 had IR. All the three patients on protease inhibitors in our ART-treated group had metabolic syndrome and IR.

Both fasting and post-glucose load blood glucose values suggest a high proportion of patients with dysglycemia, with six patients in the ART group satisfying diagnostic criteria for diabetes. In the context of high usage of nucleoside reverse transcriptase inhibitors in our ART regimes, such results appear alarming. It is to be noted here that nucleoside reverse transcriptase inhibitors used as first-line drugs in India have been strongly implicated in the development of insulin resistance and subsequent diabetes. ${ }^{16}$

The number of patients with metabolic syndrome in the ART-naïve group was three out of a total of 30. One notable difference between ART-treated and ART-naïve patients was the duration of disease. However, this bias cannot be fully eliminated as it is unethical to deny therapy to patients whose CD4 counts fall to recommended levels to commence therapy. It is also of note that, 9 out of 24 patients who had IR were not on ART, while 15 were. In addition, dysglycemia was observed in nine patients in the ART group. While mitochondrial toxicity is responsible for IR in nucleoside reverse transcriptase inhibitor-treated patients, HIV proteins Tat, Vpr, Vif, Rev, and Nef, as well as inflammatory cytokines interleukin-2 and tumor necrosis factor- $\alpha$ are implicated in ART-naïve patients. ${ }^{1}$

The BMI of our patients, both with metabolic syndrome and insulin resistance, was mostly in the range 18.5-24.9, in keeping with the lean fat Indian phenotype with central obesity. ${ }^{4}$ The ages of patients with IR and metabolic syndrome show a tendency towards younger groups, in contrast to Western studies where mean age of HIV-infected patients with these conditions is higher. ${ }^{9,10}$ The increased cardiovascular risk for South Asians with central obesity has been extensively studied. ${ }^{3,4}$ Also, the future risk for development of diabetes and cardiovascular risk in patients with metabolic syndrome is well established. ${ }^{17}$ An increased cerebro- and cardiovascular risk due to metabolic syndrome in HIV has been observed by the Data Collection on Adverse Events of Anti-HIV Drugs (DAD) study. ${ }^{2}$ However, in a risk-prone Indian population, the additional burden conferred by ART/ HIV-induced metabolic syndrome needs to be evaluated. If proven, this will have implications on future drug policies for HIV-infected patients.

Despite the limitation of the small number of patients in our study, there is an increase in metabolic syndrome, diabetes mellitus, and insulin resistance in patients on ART in southern India. Larger population-based studies are needed to assess the actual prevalence of metabolic syndrome and IR and the macrovascular risk conferred by these conditions.

\section{Disclosure}

The authors report no conflicts of interest in this work.

\section{References}

1. Leow MKS, Addy CL, Mantzoros CS. Human immunodeficiency virus/highly active retroviral therapy-associated metabolic syndromeclinical presentation, pathophysiology and therapeutic strategies. J Clin Endocrinol Metab. 2003;88(5):1961-1976.

2. d'Arminio A, Sabin CA, Phillips AN, et al; for the Writing Committee Of DAD Study Group. Cardio and cerebrovascular events in HIV infected persons. AIDS. 2004;18:1811-1817.

3. Ramachandran A, Snehalatha C, Latha E, Satyavani K, Vijay V. Clustering of cardiovascular risk factors in urban Asian Indians. Diabetes Care. 1998;21(6):967-971.

4. Yusuf S, Ounpuu S. Tackling the global epidemic of cardiovascular disease in South Asia. J Am Coll Cardiol. 2001;38(3):688-690.

5. Matthews DR, Hosker JP, Rudenski AS. Executive Summary of the Third Report of the National Cholesterol Education Program (NCEP) Expert Panel on detection, evaluation and treatment of high blood pressure in adults (Adult Treatment Panel III). JAMA. 2001;285:2486-2497.

6. Naylor BA, Treacher DF, Turner RC. Homeostatsis Model Assessment: insulin resistance and B cell function from fasting plasma glucose concentration and insulin resistance in man. Dibetologia. 1985;28: 412-419.

7. Deepa R, Shanthirani CS, Premalatha G, Sastry NG, Mohan V. Prevalence of insulin resistance syndrome in a selected south Indian population - The Chennai Urban Population Study-7 [CUPS-7]. Indian J Med Res. 2002;115:118-127.

8. American Diabetes Association. Standards of medical care in diabetes - 2009. Diabetes Care. 2009;32:S13-S61.

9. Jericó C, Knobel H, Montero M, et al. Metabolic syndrome among HIV-infected patients: prevalence, characteristics, and related factors. Diabetes Care. 2005;28:132-137.

10. Samaras K, Wand H, Law M, Emery S, Cooper D, Carr A. Prevalence of metabolic syndrome in HIV-infected patients receiving highly active antiretroviral therapy using International Diabetes Foundation and Adult Treatment Panel III criteria: associations with insulin resistance, disturbed body fat compartmentalization, elevated C-reactive protein, and [corrected] hypoadiponectinemia. Diabetes Care. 2007;30(1):113-119.

11. Deepa M, Farooqh S, Datta M, Deepa R, Mohan V. Prevalence of metabolic syndrome using WHO/ATP III and IDF definitions in Asian Indians: the Chennai Urban Rural Epidemiology Study (CURES-34). Diabetes Metab Res Rev. 2007;23:127-134.

12. Gupta R, Deedwania PC, Gupta A, Rastogi S, Panwar RB, Kothari K Prevalence of metabolic syndrome in an Indian urban population. Int J Cardiol. 2004;97:257-261. 
13. Oh J, Hegele RA. HIV-associated dyslipidaemia: pathogenesis and treatment. Lancet. 2007;7:787-796.

14. Fleishman A, Johnsen S, Systrom DM, et al. Effect of nucleoside reverse transcriptase inhibitor, stavudine on glucose disposal and mitochondrial function in muscle of healthy adults. Am J Physiol. 2007;292: E1666-E1673.

15. Antiretroviral therapy for HIV infected adults and adolescents including post-exposure prophylaxis. www.nacoonline.org
16. De Wit S, Sabin CA, Weber R, et al. Incidence and risk factors for new onset diabetes in HIV-infected patients. The data collection on adverse events of anti HIV drugs (DAD) study. Diabetes Care. 2008;31: $1224-1229$.

17. Cornier M, Dabelea D, Hernandez TL, et al. The metabolic syndrome. Endocr Rev. 2008;29(7):777-822.

\section{Publish your work in this journal}

The International Journal of General Medicine is an international, peer-reviewed open-access journal that focuses on general and internal medicine, pathogenesis, epidemiology, diagnosis, monitoring and treatment protocols. The journal is characterized by the rapid reporting of reviews, original research and clinical studies across all disease areas.
A key focus is the elucidation of disease processes and management protocols resulting in improved outcomes for the patient.The manuscript management system is completely online and includes a very quick and fair peer-review system. Visit http://www.dovepress.com/ testimonials.php to read real quotes from published authors.

Submit your manuscript here: http://www.dovepress.com/international-journal-of-general-medicine-journal 